Thomas Waechtler, Bernd Gruska, Sven Zimmermann, Stefan E. Schulz, and Thomas Gessner:

\title{
Characterization of Sputtered Ta and TaN Films by Spectroscopic Ellipsometry.
}

8th International Conference on Solid-State and Integrated Circuit Technology 2006 (ICSICT '06), Oct. 23-26, 2006, Shanghai, China. Proceedings, p. 2184-2186.

ISBN (print version): $\quad 1-4244-0160-7$

ISBN (CD-ROM version): 1-4244-0161-5

Digital Object Identifier: $\quad$ 10.1109/ICSICT.2006.306675

Available online at: $\quad$ http://ieeexplore.ieee.org/

Download and usage of this article are subject to the following terms:

This material is presented to ensure timely dissemination of scholarly and technical work. Copyright and all rights therein are retained by authors or by other copyright holders. All persons copying this information are expected to adhere to the terms and constraints invoked by each author's copyright. In most cases, these works may not be reposted without the explicit permission of the copyright holder.

(C2006 IEEE. Personal use of this material is permitted. However, permission to reprint/republish this material for advertising or promotional purposes or for creating new collective works for resale or redistribution to servers or lists, or to reuse any copyrighted component of this work in other works must be obtained from the IEEE. 


\title{
Characterization of Sputtered Ta and TaN Films by Spectroscopic Ellipsometry
}

\author{
Thomas Waechtler $^{1}{ }^{1}$, Bernd Gruska ${ }^{2}$, Sven Zimmermann ${ }^{1}$, Stefan E. Schulz ${ }^{1,3}$, Thomas Gessner ${ }^{1,3}$ \\ ${ }^{1}$ Center for Microtechnologies - ZfM, Chemnitz University of Technology, 09107 Chemnitz, Germany \\ ${ }^{2}$ SENTECH Instruments GmbH, Carl-Scheele-Strasse 16, 12489 Berlin, Germany \\ ${ }^{3}$ Fraunhofer IZM, Dept. Multi Device Integration, Reichenhainer Strasse 88, 09126 Chemnitz, Germany \\ * E-Mail: thomas.waechtler@zfm.tu-chemnitz.de
}

\begin{abstract}
Spectroscopic ellipsometry is emerging as a routine tool for in-situ and ex-situ thin-film characterization in semiconductor manufacturing. For interconnects in ULSI circuits, diffusion barriers of below $10 \mathrm{~nm}$ thickness are required and precise thickness control of the deposited layers is indispensable. In this work, we studied single films of tantalum and two stoichiometries of tantalum nitride as well as TaN/Ta film stacks both on bare and oxidized silicon. Spectroscopic ellipsometry from the UV to the NIR was applied to determine the optical properties of the films for subsequent modeling by a Lorentz-Drude approach. These models were successfully applied to TaN/Ta thin-film stacks where the values of the film thickness could be determined exactly. Moreover, it is shown that considerable differences in the optical properties arise from both film thickness and substrate.
\end{abstract}

\section{Introduction}

$\mathrm{Ta}$ and TaN thin films routinely serve as barriers against copper diffusion in metallization systems of current ultra-large scale integrated (ULSI) circuits [1, 2]. For interconnects of the future technology nodes, barrier films of far below $10 \mathrm{~nm}$ thickness are required [3]. While atomic layer deposition (ALD) is expected to replace physical vapor deposition (PVD) as the preferred deposition method, precise thickness control is indispensable for forming ultra-thin layers regardless of the technological approach.

Spectroscopic ellipsometry (SE) provides a fast and non-destructive means for both ex-situ and in-situ thin-film characterization and thickness measurement, and it is therefore emerging as a routing tool in semiconductor manufacturing. However, concise optical property data of the materials to be examined is required for modeling the SE spectra in order to exactly determine the film thickness. While data on the dielectric properties of sputtered tantalum and tantalum nitride thin films is only available over a very limited spectral range, such films were examined by SE from $190 \mathrm{~nm}$ to $2.55 \mu \mathrm{m}$ wavelength in this study. The optical properties of single films from $75 \mathrm{~nm}$ to $380 \mathrm{~nm}$ thickness were modeled by a Lorentz-Drude approach. Subsequently, film stacks of $20 \mathrm{~nm}$ TaN / $20 \mathrm{~nm}$ Ta on oxidized and non-oxidized silicon are treated using these models.

\section{Experimental}

Tantalum and tantalum nitride films from $75 \mathrm{~nm}$ to $380 \mathrm{~nm}$ thickness were prepared by reactive ion sputtering in $\mathrm{Ar}$ and $\mathrm{Ar} / \mathrm{N}_{2}$ plasmas using a Balzers Clusterline CLC9000 tool and $150 \mathrm{~mm}$ silicon wafers as substrates. Tantalum nitride layers with two different stoichiometries were obtained at Ar: $\mathrm{N}_{2}$ flow ratios of 4:1 (standard TaN - process A) and 26:1 (Ta rich, modified TaN - process B). Furthermore, film stacks of $20 \mathrm{~nm}$ standard TaN / $20 \mathrm{~nm}$ Ta were prepared in the same equipment on thermally oxidized and non-oxidized $100 \mathrm{~mm} \mathrm{Si}$ wafers.

For structural and morphological characterization of the films, the samples were examined by scanning electron microscopy (SEM), transmission electron microscopy (TEM) and electron diffraction (ED), as well as atomic force microscopy (AFM) and X-ray diffraction (XRD).

The optical characterization of the films was carried out using a SENTECH SE850 spectroscopic ellipsometer in the spectral range from $190 \mathrm{~nm}$ to $2.55 \mu \mathrm{m}$. By treating the $\Psi-\Delta$ spectra with Lorentz-Drude models, the complex dielectric function of the films was extracted. Subsequently, the models generated for single layers were applied to film stacks. In all cases, the ellipsometric data was processed using the software SpectraRay.

\section{Results and Discussion}

\subsection{Structure and Morphology}

Tantalum films of $95 \mathrm{~nm}$ to $383 \mathrm{~nm}$ thickness on Si were found to be (002) oriented $\beta-T a$, as determined by XRD. This is consistent with results reported earlier and typical for as-sputtered Ta thin films [4]. The tantalum nitride layers on $\mathrm{Si}$ were polycrystalline and strongly disordered as concluded from peak broadening in the XRD spectra. While for process $B$ mixed phases of $\mathrm{Ta}_{2} \mathrm{~N}$ and $\mathrm{Ta}_{4} \mathrm{~N}$ were grown, with process A stoichiometric TaN was obtained. SEM reveals columnar growth of the films with a lateral grain size of $24-36 \mathrm{~nm}$, as depicted by Fig. 1 (a). While the XRD spectra suggested a mixture of the hexagonal and fcc TaN phases, TEM in conjunction with ED revealed that fcc TaN was preferred. This is shown in Fig. 1 (b) and (c) where the electron diffraction 
pattern of a TaN sample is combined with the theoretical patterns to be expected for hexagonal and fcc TaN. Good agreement with the experimental result is found for the fcc phase.

The roughness of both the Ta and TaN films was determined by AFM and is in the range from 0.1 to $2.1 \mathrm{~nm}$, meaning that its influence on the ellipsometric measurements may largely be neglected.

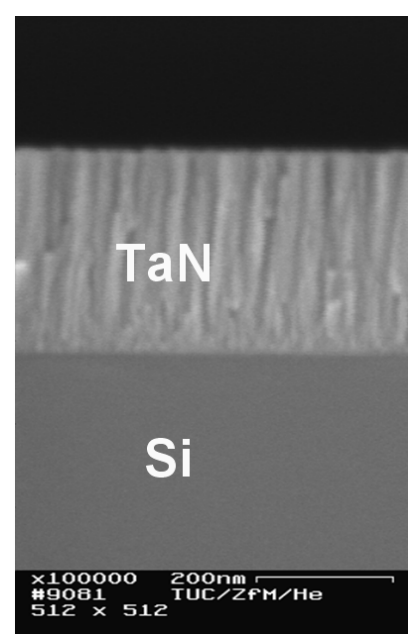

(a)

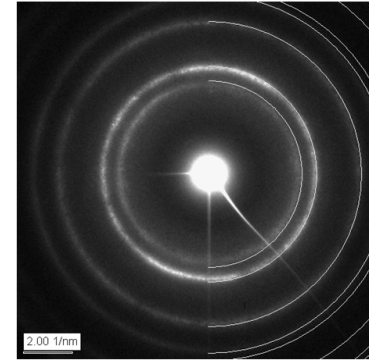

(b)

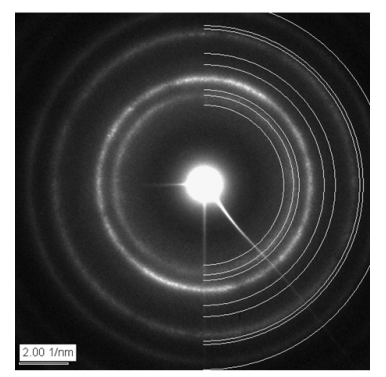

(c)
Figure 1. Cross-sectional SEM image (a) of a $290 \mathrm{~nm}$ thick TaN film on Si. The images (b) and (c) display the electron diffraction pattern of a similar sample with the theoretical rings (sharp white semicircles) for fcc TaN (b) and hexagonal TaN (c) overlaid. The good agreement of the experimental result with the theoretical pattern in case (b) suggests that the fcc TaN phase is preferred.

\subsection{Spectroscopic Ellipsometry}

To process the $\Psi-\Delta$ spectra of the films, a Lorentz-Drude oscillator model for the complex dielectric function was used $[5,6]$. For the stoichiometric TaN, a Drude term plus 3 Lorentz oscillators were sufficient to obtain reasonable fits, while a Drude term and 4 oscillators were required for the mixed phases of $\mathrm{Ta}_{2} \mathrm{~N} / \mathrm{Ta}_{4} \mathrm{~N}$. In the latter case, all films from 76 to $293 \mathrm{~nm}$ thickness were optically thick and could be treated as quasi-bulk. In case of stoichiometric TaN, the sample with a $75 \mathrm{~nm}$ film was still transparent so that the film thickness could be extracted by fitting the ellipsometry data. A value of $74.4 \mathrm{~nm}$ was obtained which agrees well with the thickness measured by cross-sectional SEM $(76.4 \mathrm{~nm})$. Fig. 3 depicts the complex refractive index of the samples showing considerable differences predominantly in the imaginary part $\mathrm{k}$. The results are similar to values reported by Aouadi and Debessai [7], while the advantage of a broad-band measurement is clearly visible.
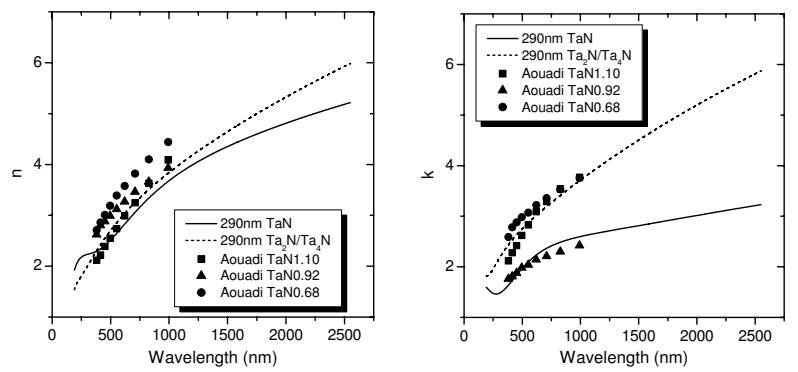

Figure 3. Optical properties of TaN films and $\mathrm{Ta}_{2} \mathrm{~N} / \mathrm{Ta}_{4} \mathrm{~N}$ samples compared with literature data [7].

Similarly, models were derived for the optical properties of the Ta films with 5 Lorentz oscillators in these cases. All samples from 95 to $383 \mathrm{~nm}$ thickness were opaque. Comparing the spectra for the complex refractive index with literature data shows good agreement with values for Ta thin films reported by Tompkins et al. [8], whereas significant differences are found for bulk Ta of the bcc phase ( $\alpha$ Ta) [9] as displayed in Fig. 4.
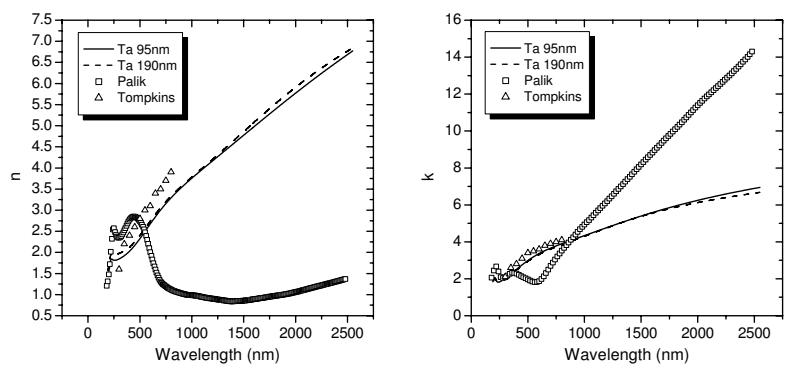

Figure 4. Optical properties of Ta films compared to data reported for similar Ta thin films (Tompkins, [8]) and bulk Ta of the $\alpha$ phase (Palik, [9]).

By applying the models derived for TaN and Ta to film stacks of $20 \mathrm{~nm} \mathrm{TaN} \mathrm{/} 20 \mathrm{~nm}$ Ta we were able to determine the thickness of the film stacks and obtain good agreement with cross-sectional SEM measurements. Fig. 5 shows the fit and the film model for a stack on silicon. While with ellipsometry the thickness of both the TaN and Ta film was determined, only the thickness of the entire stack could be measured by SEM. The error of about $9 \%$ may largely be attributed to errors of the cross-sectional SEM analysis.

In case of a TaN/Ta film stack on thermally oxidized $\mathrm{Si}(300 \mathrm{~nm} \mathrm{SiO}$ ), also the oxide thickness of $298 \mathrm{~nm}$ determined by SE somewhat deviates from the result of the SEM analysis $(284 \mathrm{~nm})$. However, it is likely that the ellipsometric value is closer to the real thickness as the thermal oxidation process is known to be rather precise.

Extracting the optical properties for the TaN/Ta films on $\mathrm{Si}$ and $\mathrm{SiO}_{2}$ reveals considerable influence of 
both film thickness and substrate. Although the Ta layer is grown on TaN in both cases, the substrate does not only influence the TaN film but has an impact on the growth of the Ta as well. Moreover, film thickness greatly affects the dielectric function, as depicted by Fig. 6.

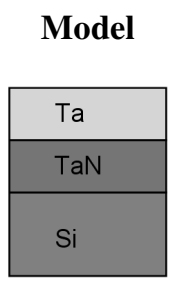

$$
\begin{array}{ll}
\text { Ellipsometry - } & \text { SEM - stack } \\
\text { thickness (nm) } & \text { thickness }(\mathbf{n m})
\end{array}
$$
22.9 $20.5\} 43.4 \quad 47.4$

Fit:
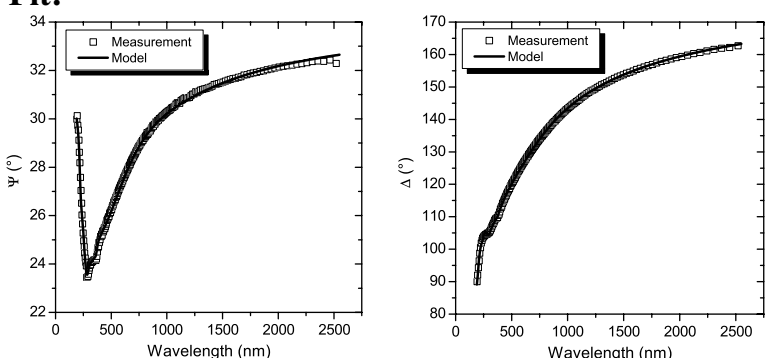

Figure 5. Ellipsometric analysis of a TaN/Ta thin film stack on Si.

However, with many fitting parameters introduced in the models, doubts may arise if the results obtained for the optical properties are real. Therefore, the SE data was tried to fit with the values of the film thickness as variable parameters only, while the oscillator models were kept as determined for the single Ta and TaN films. In this case, suitable fits were not obtained for any of the $\mathrm{TaN} / \mathrm{Ta}$ double layers. Moreover, the thickness of the stacks as well as the underlying $\mathrm{SiO}_{2}$ was determined wrong. This indicates that the results with free parameters are consistent.

\section{Summary}

Single films of $\mathrm{Ta}$, $\mathrm{TaN}$, and mixed phases of $\mathrm{Ta}_{2} \mathrm{~N} / \mathrm{Ta}_{4} \mathrm{~N}$ as well as TaN/Ta stacks on $\mathrm{Si}$ and $\mathrm{SiO}_{2}$ were examined by spectroscopic ellipsometry from $190 \mathrm{~nm}$ to $2.55 \mu \mathrm{m}$ wavelength. Good agreement of the optical properties with narrow-band data available for similar thin films was found. With Lorentz-Drude models subsequently applied to TaN/Ta double-layers the thicknesses of the film stacks could be determined in good agreement with cross-sectional SEM measurements, while the optical properties of the films strongly vary with both substrate and film thickness.

This study shows that spectroscopic ellipsometry is a valuable method for examining metallic and metal nitride thin films such as diffusion barriers required for ULSI copper metallization.
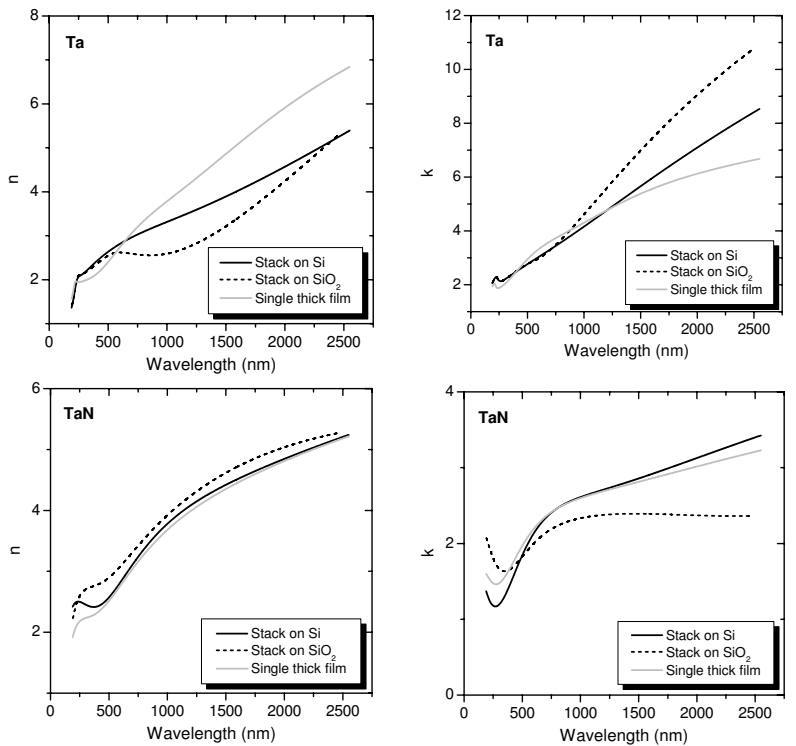

Figure 6. Optical properties of $\mathrm{Ta}$ and $\mathrm{TaN}$ films extracted for stacks on $\mathrm{SiO}_{2}$ and $\mathrm{Si}$. Considerable influence of both film thickness and substrate is visible.

\section{Acknowledgements}

The authors would like to acknowledge Steffen Schulze for TEM investigations, Andreas Pohlers for XRD analyses as well as Jürgen Bräuer and Monika Henker for AFM scans and SEM analyses.

\section{References}

[1] M.T. Wang, Y.C. Chin, and M.C. Chen, J. Electrochem. Soc. 145(7), p.2538 (1998)

[2] A.E. Kaloyeros and E. Eisenbraun, Annu. Rev. Mater. Sci. 30, p.363 (2000)

[3] SEMATECH, SIA et al. (Eds.), The International Technology Roadmap for Semiconductors (ITRS), http://public.itrs.net (2005)

[4] J. Baumann, G. Schwalbe, S. Zimmermann, C. Kaufmann, and T. Gessner, Advanced Metallization Conference 2001, p.613 (2002)

[5] H.G. Tompkins and W.A. McGahan, Spectroscopic Ellipsometry and Reflectometry, Wiley (1999)

[6] P. Patsalas and S. Logothetidis, J. Appl. Phys. 90(9), p.4725 (2001)

[7] S.M. Aouadi and M. Debessai, J. Vac. Sci. Technol. A 22(5), p.1975 (2004)

[8] H.G. Tompkins, T. Zhou and E. Chen, J. Vac. Sci. Technol. A 16(3), p.1297 (1998)

[9] E.D. Palik (Ed.), Handbook of optical constants of solids, Academic Press (1998) 